\title{
Basal cusp enlargement for repair of aortic valve insufficiency
}

\author{
Paul P. Urbanski, MD
}

\begin{abstract}
Objective: The aim of this study was to evaluate the clinical and echocardiographic results after aortic valve reconstruction with a novel surgical technique consisting of basal cusp enlargement with an autologous pericardial patch.
\end{abstract}

\begin{abstract}
Methods: Between December 2005 and June 2008, a total of 106 consecutive patients underwent elective valvesparing aortic root repair at Cardiovascular Clinic, Bad Neustadt, Germany. Fifty-nine patients required additional procedures on the aortic cusps; among them, in 10 cases basal cusp enlargement was used for restoration of coaptation area. All these patients had an ascending aortic aneurysm combined with aortic insufficiency, which was severe (4+) in 2 cases and moderate to severe $(3+)$ in 4 . The root repair was performed with valve reimplantation (David technique) in 1 case and the author's own single-patch technique in the other 9. Partial and total arch replacements were performed in 3 and 1 cases, respectively.
\end{abstract}

Results: The postoperative echocardiography at discharge showed no aortic regurgitation in 7 cases and trivial regurgitation in 3. The average coaptation height of the leaflets was $9.9 \pm 0.6 \mathrm{~mm}$, and the mean gradient across the valve was $5.4 \pm 1.9 \mathrm{~mm} \mathrm{Hg}$. At follow-up as late as 31 months, all patients were alive with echocardiographic findings unchanged from the early postoperative examinations.

Conclusions: The technique presented here allows an individualized reconstruction of the aortic cusps, leading to considerable improvement in coaptation area, in patients who have aortic leaflet prolapse or restriction caused by complex aortic root and valve disease. (J Thorac Cardiovasc Surg 2010;139:98-102)

Autologous pericardium has been used for reconstruction of the aortic valve since the early $1960 \mathrm{~s}^{1}{ }^{1}$ The pericardial patches are used mostly for cusp extensions or even replacements in rheumatic or congenital aortic valve diseases and also for the closure of cusp perforations. ${ }^{2-6}$ The technique of cusp extension in rheumatic valve disease with a pericardial patch sewn into the valve leaflet was described primarily by Bozbuga and colleagues. ${ }^{5}$ The use of a pericardial patch for repairing degenerative aortic insufficiency is not common, because this etiology often requires complex and time-consuming repair of the aortic root. Particularly in cases of aortic regurgitation caused by connective tissue disorder, complex procedures on the aortic root and valve cusps are frequently necessary. $^{7-9}$ The technique of basal cusp enlargement has been introduced in the Cardiovascular Clinic, Bad Neustadt, Germany, as an adjunct to several procedures and also used for surgery of the complex aortic root and valve diseases. The purpose of this article is to describe the technical aspects associated with this method and to report the clinical and functional results of the initial experience.

From the Cardiovascular Clinic, Bad Neustadt, Germany.

Received for publication Sept 11, 2008; revisions received Dec 17, 2008; accepted for publication March 29, 2009; available ahead of print June 11, 2009.

Address for reprints: Paul P. Urbanski, MD, Herz- und Gefaess-Klinik, Salzburger

Leite 1, 97616 Bad Neustadt, Germany (E-mail: p.urbanski@herzchirurgie.de).

$0022-5223 / \$ 36.00$

Copyright (c) 2010 by The American Association for Thoracic Surgery doi:10.1016/j.jtcvs.2009.03.058

\section{MATERIALS AND METHODS}

Between December 2005 and June 2008, a total of 115 patients at the Cardiovascular Clinic underwent valve-sparing aortic root repair (supracoronary ascending aorta replacements without root repairs excluded). One hundred six patients were operated on electively, and 56\% (59 patients) required additional procedures on the aortic cusps; among these, leaflet plication and margin reinforcement were the most frequent. In 10 patients with tricuspid aortic valves, basal cusp enlargement was used alone or in addition to several cusp procedures to improve the coaptation area of valve leaflets. Among the 10 affected patients, all had ascending aortic aneurysms of a mean diameter of $54.4 \pm 7.4 \mathrm{~mm}$, with distal extensions towards the aortic arch in 4 cases. Etiologies were degenerative, atherosclerotic, and combined in 7, 1 , and 2 patients, respectively. The detailed patient data are given in Table 1 .

\section{Surgical Technique}

Intraoperative transesophageal echocardiography was performed, according to clinic routine for all patients in whom reconstructive valve surgery is planned. The thoracic aorta and heart were exposed through a median sternotomy. For arterial return, either aortic arch or, in cases with an open arch surgery, a common carotid artery was cannulated. The technique of carotid cannulation and unilateral cerebral perfusion during circulatory arrest has been described previously. ${ }^{10}$ Myocardial protection was instituted with antegrade cold crystalloid cardioplegia. If aortic arch replacement was necessary, it was performed before aortic root repair. After the ascending aorta was opened, the aortic valve and the cause of aortic regurgitation were assessed to judge the feasibility of repair. The height of the cusps and the length of their free margins were measured with a thick yarn, which allowed simple and fast comparison of cusp dimensions. The ascending aorta was resected up to the commissures of the aortic valve. The distorted sinuses of Valsalva were excised, leaving a 2- to 3-mm rim of aortic wall attached to the aortic valve. In 4 patients, all sinuses of Valsalva had to be repaired. In the remaining 6 patients, 1 sinus ( 2 patients) or 2 sinuses (always noncoronary and right coronary) were replaced. In 
TABLE 1. Patient characteristics.

\begin{tabular}{lc}
\hline Total procedures (no.) & 10 \\
Age (y, mean \pm SD) & $61 \pm 13$ \\
Male (no.) & 8 \\
New York Heart Association functional class & 5 \\
$\quad$ III or IV (no.) & \\
Aortic insufficiency (no.) & 2 \\
$\quad$ Severe (4+) & 4 \\
$\quad$ Moderate to severe (3+) & 3 \\
Mild (2+) & 1 \\
$\quad$ Trivial (1+) & \\
Concomitant diseases (no.) & 6 \\
$\quad$ Hypertension & 3 \\
$\quad$ Coronary heart disease & 2 \\
$\quad$ Chronic obstructive pulmonary disease & \\
Logistic EuroSCORE (mean \pm SD) & $13.2 \pm 11.1$ \\
\hline
\end{tabular}

accordance with the Cardiovascular Clinic's total experience with the single-patch technique, comprising almost 200 procedures, it is possible to assert that the left coronary sinus can often be left untouched. This was the case for $60 \%$ of the patients in the current reported series. Aortic root repair thus was performed in 9 patients with the single-patch technique, ${ }^{11}$ as is routine for the Cardiovascular Clinic; however, reimplantation technique ${ }^{12,13}$ was used in 1 selected patient for demonstration purposes only. Regardless of the technique performed, a straight woven polyester graft (InterGard and InterGard Woven Aortic Thoracic Graft; InterVascular, La Ciotat, France) was used for the repairs.

If intraoperative measurement revealed an inadequate height of a cusp, basal enlargement of this cusp with an autologous pericardial patch was carried out to increase sinus depth and improve leaflet coaptation area. The patch was excised from the pericardium and fixed in a $0.625 \%$ glutaraldehyde solution for 3 minutes. Meanwhile, an incision was made directly at the base of the cusp that had to be enlarged, leaving about $5 \mathrm{~mm}$ of the leaflet toward the commissures unseparated (Figure 1, A). The patch was then trimmed to an oval form that was only few millimeters longer than the incision. The patch width was about 5 to $8 \mathrm{~mm}$, depending on the size of the coaptation deficit that had to be repaired. The patch was sewn into the leaflet with a 5-0 polypropylene continuous suture, starting at the aortic side in the middle of the incision (Figure 1, B). In patients in whom the single-patch technique for root repair was used, cusp enlargement, in addition to any other necessary procedures, was performed after completion of the root reconstruction (Figure 1,C).

In the patient operated on with the reimplantation technique, fixation of the aortic valve inside the tube was not performed until the cusp enlargement was carried out, because access to the valve cusps after reimplantation of the aortic valve into the tube was limited by the depth of the graft (Figure 1,D). After completion of the valve reimplantation inside the vascular graft, the repair was finalized by free margin reinforcement of the enlarged cusp with a continuous 6-0 suture and narrowing of the graft at the level of the sinotubular junction by placing a few additional U-stitches.

Cusp enlargement was carried out in 8 patients on the right coronary cusp and in 1 each on the left and noncoronary cusps. Mostly the procedures had to be combined with the shortening of the free margin, which was performed either by central plication or margin reinforcement with a continuous suture. This shortening was carried out on one cusp (always right coronary) in 6 patients and on two cusps (right and noncoronary) in 2 patients.

\section{RESULTS}

The mean crossclamp time, including the circulatory arrest period, was $105 \pm 30$ minutes, ranging from $50 \mathrm{~min}-$ utes to 173 minutes for a complex aortic root and valve repair and intraoperative rerepair after echocardiographic control revealed that a correction was necessary. Four patients required aortic arch repair, which was performed under circulatory arrest. In all these cases, a common carotid artery was cannulated for arterial return, and this line was used for unilateral cerebral perfusion during circulatory arrest (23 \pm 15 minutes; range, 9-70 minutes) under mild hypothermia $\left(31.0^{\circ} \mathrm{C} \pm 1.3^{\circ} \mathrm{C}\right)$. Three patients additionally required coronary revascularization for coronary heart disease.

All patients survived the surgery and were discharged from the hospital at an average of 10 postoperative days. Coumadin (warfarin sodium) was not given postoperatively except in the case of 1 patient who had undergone mechanical mitral valve prosthesis placement 8 years previously.

As mentioned previously, a second run was necessary in 1 patient after complex aortic root and valve reconstruction, with replacement of all 3 sinuses with single patches and right coronary cusp repair with basal enlargement and reinforcement of its margin with a continuous suture. During the revision, an additional central plication of the free margin was performed, which led to a successful result without any residual insufficiency. Postoperative echocardiography at discharge showed no or trivial insufficiency in 7 and 3 patients, respectively. The opening and closing movements of the aortic cusps were also favorable in all patients, regardless of the surgical method used for root repair. Most notably, no contact between the cusps and aortic wall was noted. The average coaptation height of the leaflets was $9.9 \pm 0.6 \mathrm{~mm}$, and the mean gradient across the valve was $5.4 \pm 1.9 \mathrm{~mm} \mathrm{Hg}$. During follow-up, time, which was completed for all patients and averaged $17 \pm$ 10 (range, 3-32) months, no valve-related events were noted. All patients remained alive and in New York Heart Association functional class I. The echocardiographic findings remained unchanged in all cases.

\section{DISCUSSION}

During the process of aortic root dilatation, the sinuses change in dimension in the transverse as well as longitudinal axis. Moreover, these changes are pronouncedly different in the separate sinuses. The distal movement of the commissures changes their height and also the length of the leaflet free margin, and consequently the level of cusp coaptation. For this reason, additional procedures on the valve cusps during aortic root repairs are often required, reaching the rate of $50 \%$ or more in experienced hands. ${ }^{7-9}$ On the other hand, many patients with aortic insufficiency have pathologic changes of the valve cusp and only slight dilatation of the aortic root, with a diameter of about $4 \mathrm{~cm}$ or even less. Both patients with valve pathology secondary to the root aneurysm and those with valve pathology associated 

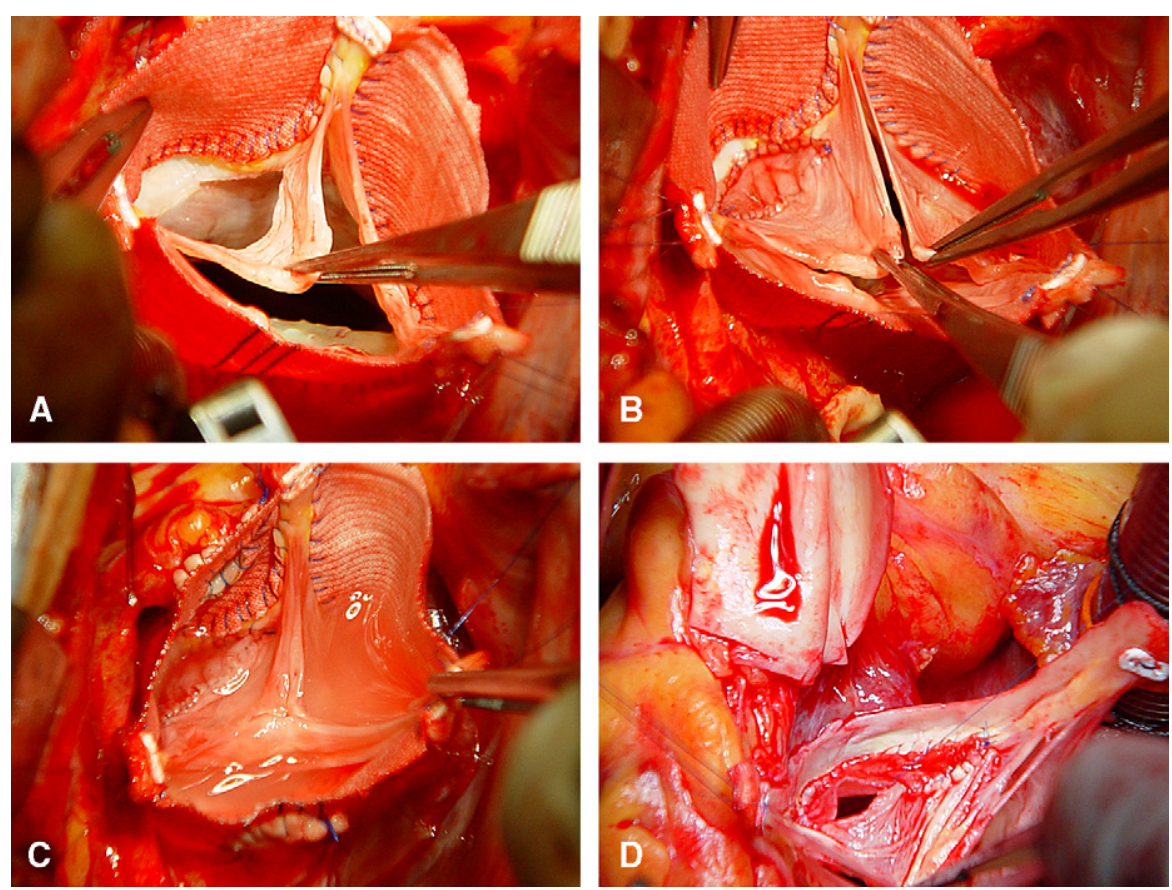

FIGURE 1. Intraoperative photographs showing basal cusp enlargement with pericardial patch in patients operated on using single patch technique (A through C) and reimplantation technique (D). A, Incision at base of cusp; B, pericardial patch sewn into cusp; C, aortic valve view after completion of cusp enlargement; D, cusp enlargement before reimplantation of valve into tube.

with only slight root dilatation often need complex surgery in which root repair is either the main procedure, frequently needing to be completed by valve repair, or an adjunct to valve repair. Particularly in the latter group, repair of all sinuses of Valsalva is seldom necessary.

Depending on the surgeon's experience and preference, the types of cusp repair vary considerably. To increase the coaptation area, many surgeons use commissural annuloplasty (commissural plication), as described originally by Cabrol and associates ${ }^{14}$; however, this procedure is only effective in correction of minor defects and is always associated with some narrowing of the aortic orifice area (Figure 2). To compensate for a larger coaptation deficit, an excessive commissural plication could be required, which can hinder the smooth leaflet motion and even cause a functional aortic stenosis. Annuloplasty is also inherent in the David aortic valve reimplantation technique, in which the entire root is reduced and the commissural plication results from subannular U-stitches. ${ }^{12}$ This method is always associated with repair of all sinuses and implantation of both coronary arteries, however, which seems, as demonstrated in the series reported here, to be unnecessary in many patients with aortic insufficiency.

An increase in the coaptation area can also be achieved by leaflet extension, in which a pericardial strip is sewn to the free edge of the cusp; however, this technique ranks among the most complex methods of aortic valve repair and is therefore rarely performed in cases with degenerative etiology necessitating aortic valve and root repair. A similar increase in sinus of Valsalva depth can be achieved by cusp enlargement with a pericardial patch sewn into the base of the valve leaflet (Figure 3). In contrast to the leaflet edge extension, in
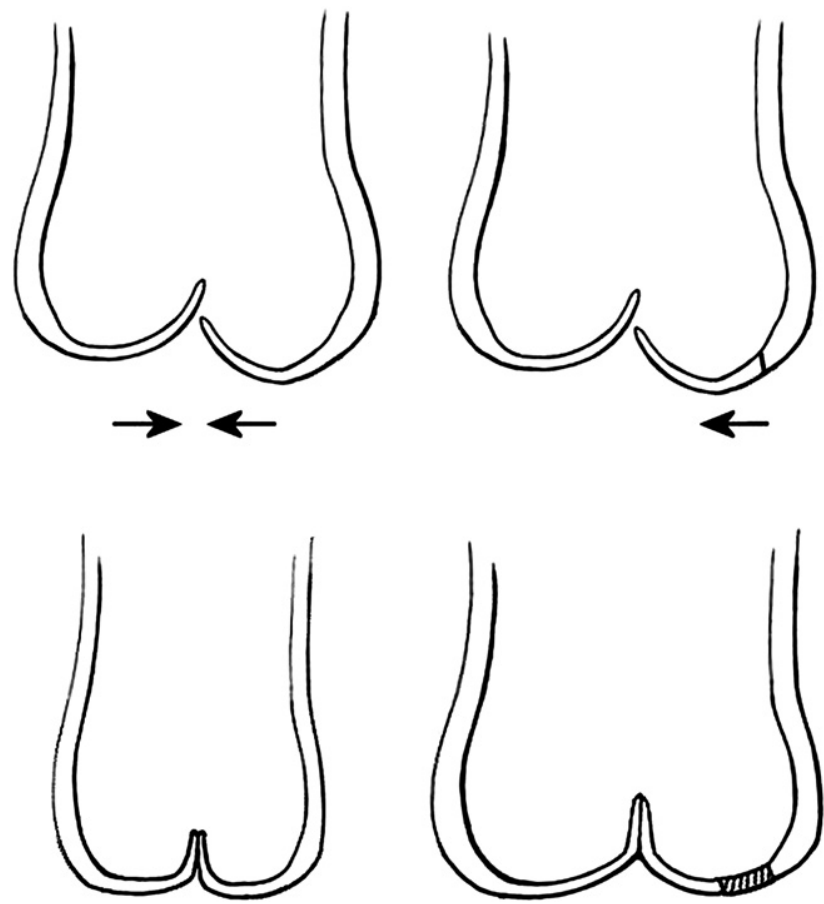

FIGURE 2. Schematic illustration of aortic root showing restoration of valve coaptation by narrowing subcommissural triangle (left) or increasing sinus depth withal cusp enlargement (right). 

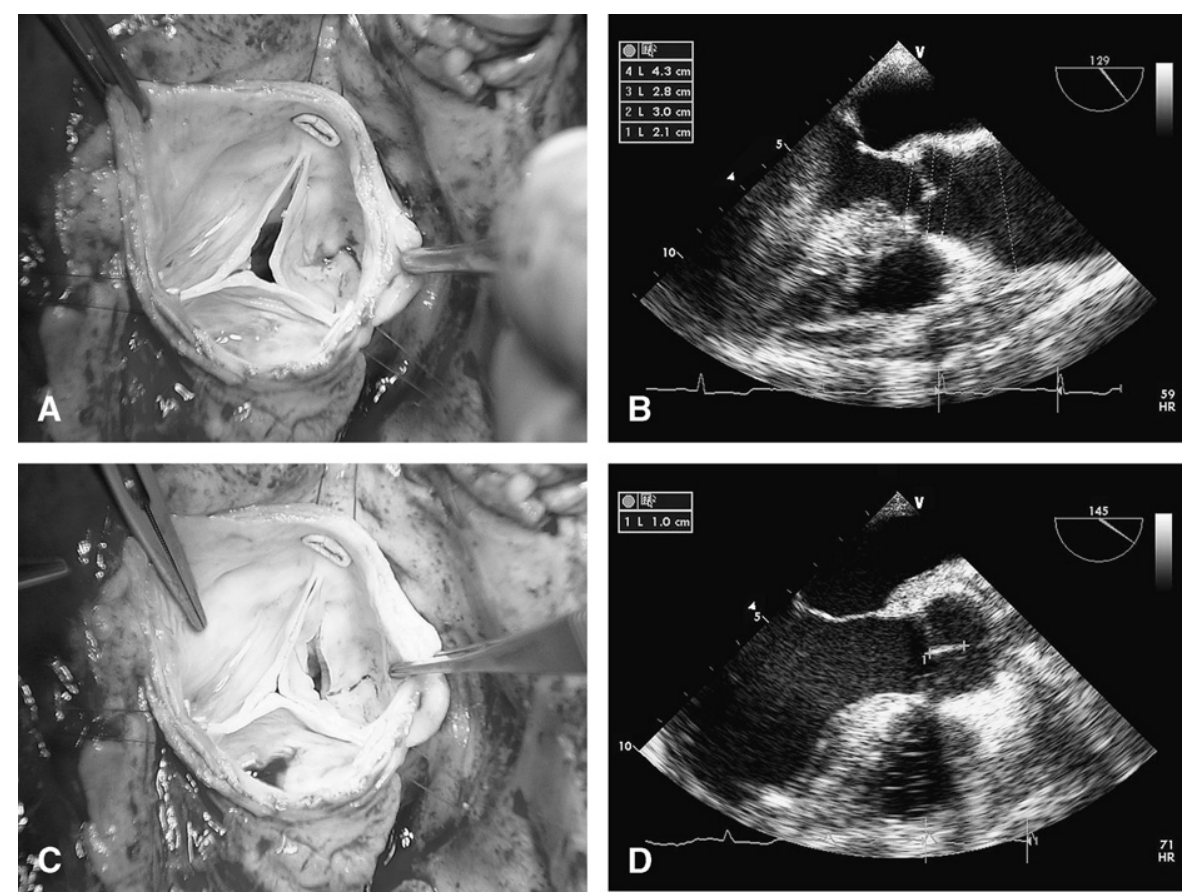

FIGURE 3. Operative photograph (A) and preoperative transesophageal echocardiogram (B) showing aortic aneurysm with moderate dilatation of right coronary sinus of Valsalva and restriction of noncoronary cusp. C, Operative photograph showing good coaptation of all cusps after incision at base of restricted leaflet and its centripetal movement. D, Postoperative transesophageal echocardiogram showing excellent coaptation area with length of $10 \mathrm{~mm}$ after basal enlargement of noncoronary cusp with pericardial patch, repair of right coronary sinus of Valsalva with single-patch technique, and supracoronary ascending aorta replacement.

which commissural anchoring of the pericardial strip and the determination of the proper length of its free margin pose a surgical challenge, a patch enlargement of the cusp at its base is much easier. The correction of the free margin of the native cusp (eg, by reinforcement or plication), if it is even necessary after basal enlargement, is also easier than the recreation of a completely new cusp edge. Moreover, the coaptation surface is created by the leaflet tissue rather than by pericardial material and a suture line. This is a possible explanation for the results reported by Minakata and associates ${ }^{15}$ showing that patients with aortic valve insufficiency who undergo cusp repair with patch plasty for perforations have the best long-term results among all patients operated on with various surgical techniques. This aspect seems to be correlated with the patch plasty described in which the basal enlargement with pericardial patch can be compared with closure of the perforation.

Although it is theoretically possible for multiple cusps to be enlarged, in all 10 patients in this series, enlargement of only a single cusp was required. In $80 \%$ of the patients, this was the right coronary cusp, which supports the previous anatomic findings revealing that the right cusp has a slightly longer free margin and less depth than the left or noncoronary cusp. ${ }^{16}$

In all cases, regardless of the technique used for the aortic root repair or the number of sinuses replaced, the method described led to considerable improvement in the leaflet coaptation surface and excellent early and midterm functional results. I believe that this technique is an interesting option for cusp repair in patients who have aortic leaflet prolapse or restriction caused by complex aortic root and valve disease. It can enrich our armamentarium for surgical treatment of these pathologies.

I thank Mrs Melissa Lindner, Mrs Alexandra Metz, and Mrs Bianca Müller for their assistance in preparing this article.

\section{References}

1. Ross DN. Surgical reconstruction of the aortic valve. Lancet. 1963;1:571-4.

2. Duran C, Kumar N, Gometza B, Halees Z. Indications and limitations of aortic valve reconstruction. Ann Thorac Surg. 1991;52:447-54.

3. Ahn H, Kim KH, Kim YJ. Midterm result of leaflet extension technique in aortic regurgitation. Eur J Cardiothorac Surg. 2002;21:465-9.

4. Grinda JM, Latremouille C, Berrebi A, Zegdi R, Chauvaud S, Carpentier AF, et al. Aortic cusp extension valvuloplasty for rheumatic aortic valve disease: midterm results. Ann Thorac Surg. 2002;74:438-43.

5. Bozbuga N, Erentug V, Kirali K, Akinci E, Isik Ö, Yakut C. Midterm results of aortic valve repair with the pericardial cusp extension technique in rheumatic valve disease. Ann Thorac Surg. 2004;77:1272-6.

6. Lausberg H, Aicher D, Langer F, Schäfers HJ. Aortic valve repair with autologous pericardial patch. Eur J Cardiothorac Surg. 2006;30:244-9.

7. Jeanmart H, De Kerchove L, Glineur D, Goffinet JM, Rougui I, Van Dyck M, et al. Aortic valve repair: the functional approach to leaflet prolapse and valvesparing surgery. Ann Thorac Surg. 2007;83:S746-51.

8. Aicher D, Langer F, Adam O, Tscholl D, Lausberg H, Schäfers H-J. Cusp repair in aortic valve reconstruction: does the technique affect stability? J Thorac Cardiovasc Surg. 2007;134:1533-9. 
9. Urbanski P, Zhan X, Frank S, Diegeler A. Aortic root reconstruction using new vascular graft. Interact Cardiovasc Thorac Surg. 2009;8:187-90.

10. Urbanski P, Lenos A, Lindemann Y, Weigang E, Zacher M, Diegeler A. Carotid artery cannulation in aortic surgery. J Thorac Cardiovasc Surg. 2006;132: 1398-403.

11. Urbanski P. Valve-sparing aortic root repair with patch technique. Ann Thorac Surg. 2005;80:839-44.

12. David TE, Feindel CM. An aortic valve-sparing operation for patients with aortic incompetence and aneurysm of the ascending aorta. $J$ Thorac Cardiovasc Surg. 1992;103:617-22.
13. Urbanski PP, Frank S. New vascular graft for simplification of the aortic valve reimplantation technique. Interact Cardiovasc Thorac Surg. 2008;7:552-5.

14. Cabrol C, Cabrol A, Guiraudon G, Bertrand M. [Treatment of aortic insufficiency by means of aortic annuloplasty] [Article in French]. Arch Mal Coeur Vaiss. 1966; 59:1305-12.

15. Minakata K, Schaff H, Zehr K, Dearani J, Daly R, Orszulak T, et al. Is repair of aortic valve regurgitation a safe alternative to valve replacement? J Thorac Cardiovasc Surg. 2004;127:645-53.

16. Vollebergh FE, Becker AE. Minor congenital variations of cusp size in tricuspid aortic valves. Possible link with isolated aortic stenosis. Br Heart J. 1977;39:1006-11. 